University of Nebraska - Lincoln

DigitalCommons@University of Nebraska - Lincoln

\title{
Supporting Narrative Retells for People With Aphasia Using Augmentative and Alternative Communication: Photographs or Line Drawings? Text or No Text?
}

Julie Griffith

University of Cincinnati, julie.griffith@uc.edu

Aimee R. Dietz

University of Cincinnati, aimee.dietz@uc.edu

Kristy S.E. Weissling

University of Nebraska - Lincoln, kristy.weissling@unl.edu

Follow this and additional works at: https://digitalcommons.unl.edu/specedfacpub

Part of the Analytical, Diagnostic and Therapeutic Techniques and Equipment Commons, Special Education and Teaching Commons, Speech and Hearing Science Commons, and the Speech Pathology and Audiology Commons

Griffith, Julie; Dietz, Aimee R.; and Weissling, Kristy S.E., "Supporting Narrative Retells for People With Aphasia Using Augmentative and Alternative Communication: Photographs or Line Drawings? Text or No Text?" (2014). Special Education and Communication Disorders Faculty Publications. 153.

https://digitalcommons.unl.edu/specedfacpub/153

This Article is brought to you for free and open access by the Department of Special Education and Communication Disorders at DigitalCommons@University of Nebraska - Lincoln. It has been accepted for inclusion in Special Education and Communication Disorders Faculty Publications by an authorized administrator of DigitalCommons@University of Nebraska - Lincoln. 


\title{
Supporting Narrative Retells for People With Aphasia Using Augmentative and Alternative Communication: Photographs or Line Drawings? Text or No Text?
}

\author{
Julie Griffith, ${ }^{1}$ Aimee Dietz, ${ }^{1}$ and Kristy Weissling ${ }^{2}$ \\ 1 University of Cincinnati \\ 2 University of Nebraska-Lincoln \\ Corresponding author — Julie Griffith, griff2jd@mail.uc.edu
}

\begin{abstract}
Purpose: The purpose of this study was to examine how the interface design of an augmentative and alternative communication (AAC) device influences the communication behaviors of people with aphasia during a narrative retell task.

Method: A case-series design was used. Four narratives were created on an AAC device with combinations of personally relevant (PR) photographs, line drawings (LDs), and text for each participant. The narrative retells were analyzed to describe the expressive modality units (EMUs) used, trouble sources experienced, and whether trouble sources were repaired. The researchers also explored the participants' perceived helpfulness of the interface features.

Results: The participants primarily used spoken EMUs to retell their narratives. They relied on PR photographs more frequently than LDs; however, they reported both picture types to be equally helpful. Text was frequently used and reported as helpful by all 4 people with aphasia. Participants experienced similar rates of trouble sources across conditions; however, they displayed unique trends for successful repairs of trouble sources.

Conclusion: For narrative retells, LDs may serve as an effective visual support when PR photographs are unavailable. Individual assessment is necessary to determine the optimum combination of supports in AAC systems for people with aphasia.
\end{abstract}

Keywords: aphasia, augmentative and alternative communication, visual supports, text, personally relevant

Technology is an integral part of daily life (Brandenburg, Worrall, Rodriguez, \& Copland, 2013); as such, proficient computer and communication skills are essential to maintain employment and a social community following stroke (Dietz, Ball, \& Griffith, 2011; Elman \& Larsen, 2010). Many people with aphasia have expressed interest in using computers to augment their communication (True, Bartlett, Fink, Linebarger, \& Schwartz, 2010), for word processing, e-mail, and online shopping (Elman \& Larsen, 2010). However, increasing evidence suggests that the interface design of high-technology augmentative and alternative communication (AAC) devices plays an important role in the proficiency and communicative success experienced by people with aphasia (e.g., Dietz, Weissling, Griffith, \& McKelvey, 2012; Wallace \& Hux, 2014). Therefore, how elements of interface design affect accessibility and communication needs to be examined.

Historically, high-technology AAC devices were designed for people with relatively intact language skills, such as people with cerebral palsy (Dietz, Beukelman, \& McKelvey, 2006). Traditional interfaces on high-technology AAC devices use a gridbased layout, which require users to rely largely on linguistic processing to generate messages. For example, to create a sentence, people with aphasia must combine single icons with their associated text labels and then sequence the icons in a syntactically appropriate manner. Although people with aphasia are able to learn to use these interfaces, the process is often lengthy (e.g., Fox, Sohlberg, \& Fried-Oken, 2001; Koul, Corwin, \& Hayes, 2005). In an effort to reduce the time required to learn AAC interfaces, researchers have begun to examine ways to display information on AAC devices that reduce the amount of linguistic processing required for successful use.

Visual scene displays (VSDs) for people with aphasia were developed to enhance communication by providing supports that take advantage of the relatively intact visual- spatial skills and autobiographical memory of people with aphasia (Dietz et al., 2006; McKelvey, Dietz, Hux, Weissling, \& Beukelman, 
2007). High-technology VSDs typically include personally relevant (PR) photographs, text, and digitized speech (i.e., speak buttons), all of which aid communicative success (for a detailed overview of VSDs see Dietz et al., 2006, 2012; McKelvey et al., 2007; Wallace \& Hux, 2014). Results of these preliminary studies indicate that people with aphasia quickly learn navigation and message representation strategies using VSDs. Yet, the knowledge of the specific elements that facilitate the most effective use of VSDs by people with aphasia is just beginning to unfold. Therefore, the examination of specific features of VSDs, such as types of visual supports and the inclusion of text, is important to the development of effective AAC systems for people with aphasia.

\section{Visual Supports}

Visual supports may take several forms, such as PR photographs, nonpersonally relevant photographs, and line drawings (LDs; Wallace, Dietz, Hux, \& Weissling, 2012). A PR photograph is an image "that connects in some way to the person showing it or viewing it: Either the person is in the photograph, or the individuals and setting depicted are highly familiar to that person" (McKelvey, Hux, Dietz, \& Beukelman, 2010, p. 23).Nonpersonally relevant photographs convey similar information as PR photographs; however, they differ because the people and context depicted are not familiar to the person viewing it (McKelvey et al., 2010). Dietz et al. (2012) reported a case series that described how four people with aphasia retold narratives using four VSD interfaces that included both PR photographs and nonpersonally relevant photographs with and without accompanying text. The findings suggest that the integration of PR photographs generated more efficient narrative retells for the participants when compared with interface designs that displayed nonpersonally relevant photographs. In particular, the two people with aphasia who had no prior AAC experience did not demonstrate any trouble sources in the retell that displayed PR photographs and text. Further, during the retell that used PR photographs without text, they resolved trouble sources more effectively than during the retells with nonpersonally relevant photographs. In addition, despite forewarning that several of the narratives would be displayed with nonpersonally relevant photographs, all of the participants experienced some degree of off-topic commentary and spent relatively large proportions of time explaining to a communication partner that the photographs were not their own. Further, the participants reported a perceived increased benefit from the presence of PR over nonpersonally relevant photographs, strengthening the argument to use PR materials during narrative retell tasks.

Although technological advances have made the incorporation of PR photographs onto devices effortless, people do not always have PR photographs accessible for every retell topic they wish to discuss. This finding warrants the examination of acceptable alternatives for when PR photographs are not available; one option is the inclusion of LDs. Line drawings are inherently nonpersonally relevant in that they are computergenerated symbols, which convey ideas and objects. Wallace et al. (2012) described LDs as images that "are created with writing or painting utensils or graphic design software; they can be black-and-white or color images with shading being optional" (p. 165). Traditionally, LDs are used as a form of symbol-based communication (e.g., Boardmaker) and are frequently preloaded on high-technology AAC devices. Yet, it is believed that photographs enhance gestalt comprehension more easily than LDs (Beukelman \& Mirenda, 2013; Dietz, Hux, McKelvey, Beukelman, \& Weissling, 2009). Because aphasia is conceptualized as a symbolic-processing deficit (McNeil \& Pratt, 2001), communicative intervention strategies that reduce the amount of symbolic processing people with aphasia need to perform may increase the communicative success they experience. However, how well specific types of visual supports (e.g., PR photographs, nonpersonally relevant photographs, color LDs) facilitate successful communication is largely unknown.

\section{Linguistic Supports}

In a recent study, people with aphasia and their communication partner identified text as an important element to include on high-technology VSDs (Dietz et al., 2012). In particular, the people with aphasia perceived the presence of text as very helpful when retelling personal narratives. In this study, the communication partner reported that text was integral to her understanding of the participants' narrative; in fact, she questioned her ability to fully understand the narrative retells when text was not present. These findings extend a small body of literature that documents the success of using text to facilitate improved communication (e.g., Dietz et al., 2006; Garrett \& Beukelman, 1995; Smith \& Garrett, 2005); however, additional studies are necessary to provide further guidance on how people with aphasia use and perceive text across a variety of interface designs.

Although the extant literature includes several single-subject design and case-series examinations regarding how various elements of VSD interfaces influence the communicative behaviors of people with aphasia, questions remain regarding how the type of visual supports (e.g., PR photographs or LDs) and the presence of linguistic supports (i.e., text boxes or no text boxes) influence the communication of people with aphasia. Therefore, the purpose of this investigation was to describe the (a) expressive modalities used, (b) trouble sources experienced, (c) patterns in repair of trouble sources, (d) perceived helpfulness of visual and linguistic supports, and (e) perceived helpfulness of the high-tech AAC VSD device when four people with aphasia retold personal narratives using four variants of a high-technology AAC VSD interface. The four VSD interfaces created for this investigation included: (a) PR photographs with text boxes (PR + TB), (b) PR photographs without text boxes (PR NO TB), (c) LDs with text boxes (LD + TB), and (d) LDs without text boxes (LD NO TB; see Figure 1). 
a)

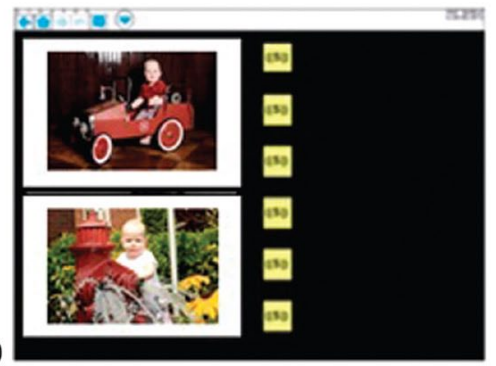

c)

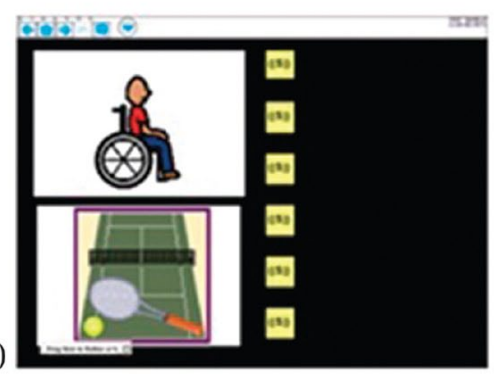

b)

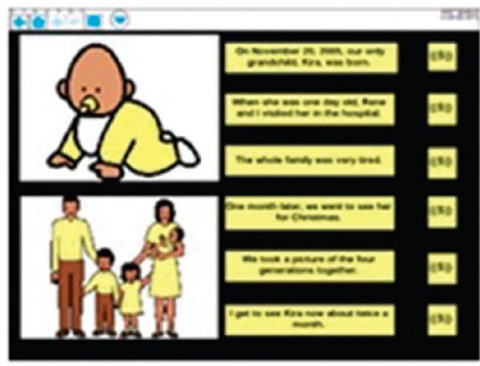

d)

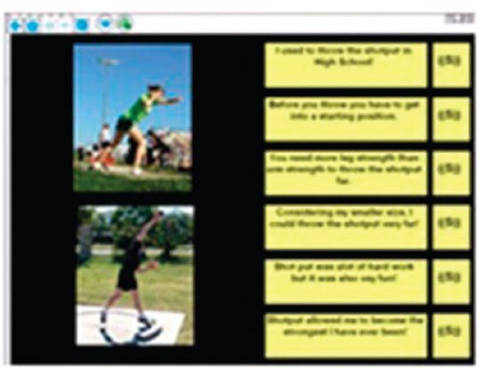

(C)2012 Dynavox Mayer-Johnson. All rights reserved.

Figure 1. Examples of the four experimental visual scene displays. (a) personally relevant (PR) photographs without text boxes (PR NO TB), (b) line drawings (LDs) with text boxes (LD + TB), (c) LDs without text boxes (LD NO TB), and (d) PR photographs with text boxes (PR + TB).

\section{Method}

\section{Research Design}

This investigation aimed to describe how the presence of visual and linguistic supports influenced the communicative behaviors of four people with aphasia and explore their perceived helpfulness of these supports. Therefore, we used a case-series approach to allow the researchers to discover patterns of communicative behavior unique to individuals that may otherwise be overlooked in group study designs (Creswell, 2009).

\section{Participants}

A convenience sampling method yielded four participants with chronic, moderate-severe Broca's aphasia as classified by a Western Aphasia Battery-Revised (WAB-R) Aphasia Quotient (AQ; Kertesz, 2007; $M=53.17$, range = 32.1-64.9). The researchers determined the participants' reading comprehension ability by using the supplemental reading comprehension of sentences subtest of the WAB-R, which revealed a large range $(M=25$, range $=2-40)$. All participants were Caucasian and shared the following characteristics: (a) had aphasia because of a left cerebrovascular accident (CVA), (b) were right-handed before onset of aphasia, (c) were at least 12 months poststroke $(M=56.5$, range $=42-81),(d)$ were medically stable, $(\mathrm{e})$ had a negative history of major psychotic episodes or substance abuse, (f) had vision and hearing within functional limits, and (g) were native speakers of American English. Detailed participant profiles are provided in the Results section. We display a summary of their demographic information and linguistic assessment performance in Table 1.

\section{Equipment}

The researchers created the VSDs on a DynaVox Vmax ${ }^{\mathrm{TM}}$, using narratives constructed with the participants. Mayer-Johnson Boardmaker $®$ software allowed creation of the LDs. Three digital video cameras (Canon FS200) captured (a) spoken language, facial expressions, and gestures; (b) activity on the device; and (c) written and drawn data during the narrative retell session, all of which were used for later transcription.

\section{Procedures}

Linguistic testing. The participants provided consent and completed two linguistic testing sessions to minimize effects of fatigue. To document aphasia type and severity, the participants completed the WAB-R. The reading comprehension of sentences subtest of the WAB-R (Kertesz, 2007) was also given to document the participants' basic reading skills. Six weeks elapsed from the time of consent to the narrative retell session for each participant. This large time span was primarily because of the lengthy narrative development period.

Narrative development. Each participant supplied personal photographs that were used to develop six narratives during two co-construction sessions (Dietz et al., 2006) with the first author. All of the narratives provided by the participants focused on family, vacations, and significant life events (e.g., first day of school). The people with aphasia verified the accuracy of all the narratives with the first author and decided on the 
Table 1. Participant demographic and language measures.

\begin{tabular}{|c|c|c|c|c|c|c|c|c|c|}
\hline Participant & $\begin{array}{l}\text { Age } \\
\text { (yrs) }\end{array}$ & Gender & $\begin{array}{l}\text { Education } \\
\text { level }\end{array}$ & Ethnicity & $\begin{array}{l}\text { Months } \\
\text { post onset }\end{array}$ & $\begin{array}{l}\text { High-technology } \\
\text { AAC experience }\end{array}$ & $\begin{array}{l}\text { Aphasia } \\
\text { type }\end{array}$ & $\begin{array}{c}\text { WAB-R } \\
\mathrm{AQ}^{\mathrm{a}}\end{array}$ & $\begin{array}{l}\text { WAB-R Reading } \\
\text { comprehension } \\
\text { of sentences }^{b}\end{array}$ \\
\hline Jack & 57 & Male & Bachelors' degree & Caucasian & 48 & No & Broca's & 61.8 & 28 \\
\hline Sarah & 42 & Female & Bachelors' degree & Caucasian & 81 & Yes & Broca's & $53.9^{d}$ & 2 \\
\hline
\end{tabular}

AAC = augmentative and alternative communication. $\mathrm{a}$. WAB-R AQ $=$ Western Aphasia Battery-Revised Aphasia Quotient, maximum score $=100$. b. maximum score $=40$ (Kertesz, 2007). c. concomitant dysarthria. d. concomitant apraxia of speech.

wording included in the six text boxes and speak buttons $(M=$ 68 words per narrative, $M=11.5$ words per sentence, $M=4$ th grade Flesch-Kincaid grade level). The first author prompted the participants to choose their four favorite narratives to retell to a naive listener by telling them to "Choose four stories to tell to someone new to you."

The researchers randomly assigned the four selected narratives to one of the four experimental VSD designs and programmed the $\operatorname{Vmax}^{\mathrm{TM}}$ with color PR photographs supplied by the participants or colored LDs. The researchers adapted procedures described by McKelvey et al. (2010) to create the LDs. This was accomplished by conducting a key word search in the Boardmaker image database. For example, to match a PR photograph of a daughter's wedding, the key words wedding and bride were used in the search. Three naive raters evaluated the top three hits produced from this key word search using a 5-point Likert scale (i.e., 1 = strongly disagree; 3 = neutral; 5 = strongly agree that the $L D$ resembles the PR photograph). If a key word search did not yield an appropriate LD (i.e., an average rating of at least 3), the first author combined available Boardmaker images to create a new image. This process continued until a rating of 3 was achieved, indicating that the LD objectively resembled the PR photograph. All LDs achieved a minimum average rating of 3 or higher $(M=3.35, S D=0.14)$.

Orientation session. Prior to the narrative retell sessions, the first author familiarized the people with aphasia with the AAC device and the four variants of VSDs. During this period, the first author pointed out the various features of the interfaces (i.e., pictures, text boxes, and speak buttons). After the interface orientation, the participants explored the interface for a few minutes. The researcher also explained that they would retell narratives with and without text boxes, and the narratives would include either PR photographs or LDs. During this description, the researcher displayed an example of each interface type using two personal narratives that were not selected for use during the retell task (see Figure 1). Procedural integrity checks revealed that the researcher followed the familiarization protocol with $100 \%$ accuracy.

Narrative retell session. Prior to retelling the four narratives, the first author introduced the listener to the person with aphasia to allow the pair to become comfortable with each other.
At an appropriate time after the introduction, the first author turned the Vmax to the first target narrative. In between each retell, the first author excused the listener and asked the participants several questions about their perceived helpfulness of the interface. The narratives were presented in random order during a single session to minimize order effects.

The naive listener was a 20-year-old undergraduate female in communication sciences and disorders blinded to the purpose of this study. Before this investigation, she had limited experience interacting with people with aphasia. Guidelines for the listener's interactions with the participants were established. The listener was trained to (a) begin the retell with, "I understand you want to talk to me about__."; (b) provide pause time; (c) ask open-ended questions; (d) ask for confirmation or correctness if needed; and (e) not to use vocabulary related to the topic unless previously provided by the participant (Dietz et al., 2012). The researcher reviewed the guidelines with the listener before each narrative retell session. Procedural integrity checks revealed that the listener followed the guidelines $95 \%$ of all opportunities.

Informal interviews. On completion of each retell, the people with aphasia answered two Likert scale questions regarding their perceived helpfulness of the visual and linguistic supports made available to them (1 = strongly disagree; 5 = strongly agree that the words/pictures were helpful ). In addition, they rated the helpfulness the AAC device during the retell $(1=$ not helpful; 3 = very helpful). Also, on the basis of their responses to the Likert scale questions, the first author engaged the people with aphasia in a brief conversation about their experiences. Quotes were extracted from the conversations to reflect the participants' opinions.

\section{Data Analyses}

The narrative retell sessions were transcribed verbatim and were divided into expressive modality units (EMUs). The types of EMUs coded were spoken, picture (i.e., photograph or LD), text box, speak button, written, and drawn. Expressive modality units were defined as containing a single thought or idea conveyed through any modality such as speech, reference to pictures or text, writing, and drawing (Dietz et al., 2012). The participants were allowed to talk as long as they deemed necessary 
to retell each narrative. As such, EMU usage was calculated as a percentage of the total number of EMUs expressed. The transcripts were also analyzed for trouble sources and percentage of repaired trouble sources. Trouble sources were defined as a lack of information provided in the EMU that impeded the transition or flow of the interaction, which prompted the participant to give or the listener to request more information (Dietz et al., 2012). A repair was considered successful when the flow of the interaction was restored. For example, a trouble source was repaired when the listener restated her interpretation of the repaired content, the person with aphasia confirmed the information, and the dyad moved onto the next topic. In addition, the trajectory of trouble sources or the average number of EMUs required to resolve a trouble source was measured. Interrater reliability for each dependent measure was at least $80 \%$.

\section{Results}

The following sections summarize the EMUs used and trouble sources experienced and repaired by the participants for each VSD condition. In addition, the participants' perceived helpfulness of the visual and linguistic supports as well as the AAC device is reported.

\section{Ellen}

At the time of this study, Ellen was a 64-year-old female who was 55 months poststroke. She was a retired social worker and enjoyed time with her family, especially her grandchildren and husband. Ellen received physical, occupational, and speech-language therapy services previously; she was not enrolled in a rehabilitation program during this investigation. Further, her rehabilitation experience did not include high-technology AAC. Ellen exhibited severe Broca's aphasia (i.e., WAB-R AQ = 32.1; Kertesz, 2007) and concomitant moderate dysarthria. She primarily communicated through the use of residual speech; her verbal output was characterized by reduced phrase length and word retrieval (e.g., 2-4 words), whole word and phrase perseverations, and semantic paraphasias.

Ellen's use of the available EMUs across the four conditions is provided in Figure 2. In Figure 3, we summarize Ellen's trouble sources and repairs. In Table 2, we display Ellen's perceived helpfulness of the visual and linguistic supports. Of note, at the end of her final retell, Ellen rated the AAC device as very helpful when retelling her narratives (Table 2).

PR photographs with text boxes. Ellen used a variety of EMUs to retell her narrative in this condition. Spoken EMUs comprised the majority of EMUs (64\% of the total EMUs); however, she also used text box (21\% of the total EMUs), picture (12\% of the total EMUs), and speak button EMUs (3\% of the total EMUs). She experienced trouble sources on $10 \%$ of her EMUs, of which $80 \%$ were repaired with an average trajectory of 7 EMUs. Ellen agreed the PR photographs were helpful (i.e., 4) and strongly agreed text helped her tell her story (i.e., 5).

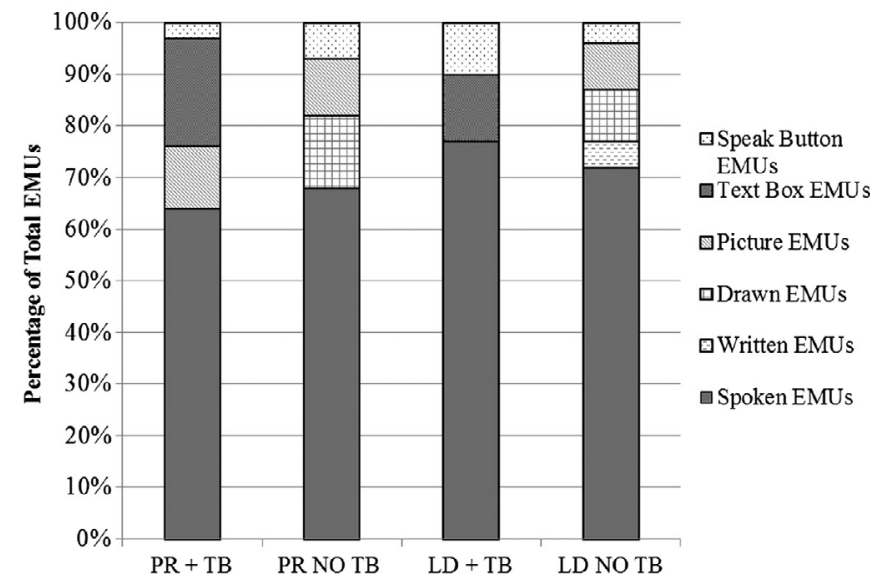

Figure 2. The percentage of expressive modality types out of the total expressive modality units Ellen used during each narrative retell condition. $\mathrm{EMU}=$ expressive modality unit.

PR photographs without text boxes. The majority of Ellen's EMUs were spoken (68\% of total EMUs). She also used drawn (14\% of total EMUs), picture (11\% of total EMUs), and speak button EMUs (7\% of total EMUs) to retell her narrative. Trouble sources comprised $9 \%$ of her total EMUs, of which $50 \%$ were repaired with an average trajectory of 10 EMUs. Ellen strongly agreed (i.e., 5) that the words would have been helpful when telling her story. Ellen disagreed (i.e., 2) that the PR photographs were helpful when telling her story in this condition. However, when Ellen was asked to clarify her rating of the PR pictures she conveyed that more pictures would have been helpful in the succeeding interaction:

Researcher: So the pictures helped a little bit [pointing to the PR NO TB interface]?

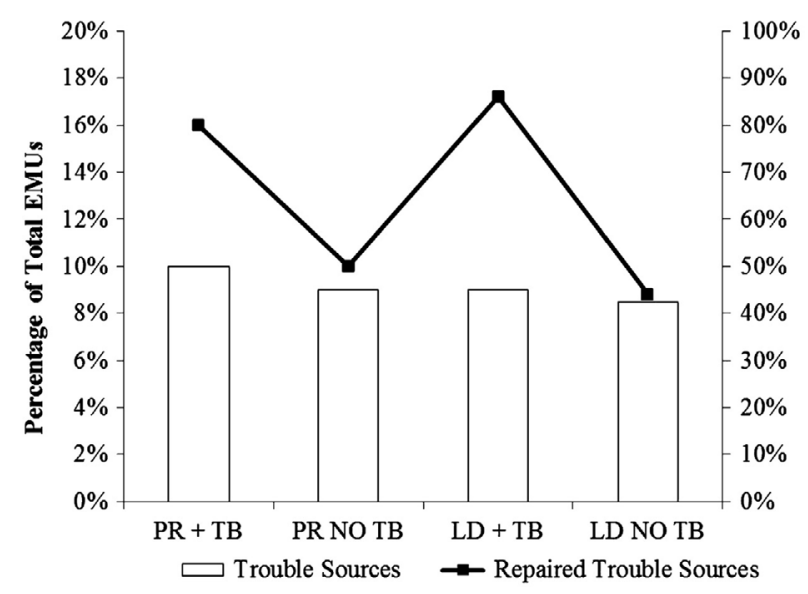

Figure 3. The percentage of trouble sources Ellen experienced out of the total amount of EMUs in each conditions as well as the percentage of trouble sources Ellen was able to repair. 
Table 2. Participants' perceived helpfulness of the photographs, line drawings, text, and AAC device.

\begin{tabular}{|c|c|c|c|c|c|c|c|c|c|}
\hline \multirow[b]{2}{*}{ Participant } & \multicolumn{4}{|c|}{$\begin{array}{l}\text { The pictures helped me } \\
\text { tell the story. }\end{array}$} & \multicolumn{4}{|c|}{$\begin{array}{l}\text { The words helped/would have } \\
\text { helped me tell the story. }\end{array}$} & \multirow{2}{*}{$\begin{array}{c}\text { How helpful was the } \\
\text { computer (AAC device)? } \\
\text { Overall rating }\end{array}$} \\
\hline & $\mathrm{PR}+\mathrm{TB}$ & PR NO TB & $\mathrm{LD}+\mathrm{TB}$ & LD NO TB & $P R+T B$ & PR NO TB & $L D+T B$ & LD NO TB & \\
\hline Ellen & 4 & 2 & 5 & 3 & 5 & 5 & 4 & 5 & 3 \\
\hline Jack & 4 & 5 & 5 & 4 & 5 & 4 & 5 & 4 & 2 \\
\hline
\end{tabular}

$P R+T B=$ personally relevant $(P R)$ photographs with text boxes; PR NO TB $=$ PR photographs without text boxes; $L D+T B=$ line drawings $(L D s)$ with text boxes; $L D$ NO TB $=$ LDs without text boxes. a: $1=$ strongly disagree; $5=$ strongly agree. $b: 1=$ not helpful; $2=$ helpful; $3=$ very helpful.

Ellen: Yes. What uh mmmo:02 sec [pointing at the two pictures displayed on the VSD and makes a scrolling motion with her hand]?

Researcher: So you feel like you needed more pictures to tell your story?

Ellen: Yes.

$L D$ with text boxes. During the LD + TB condition, Ellen relied mostly on spoken EMUs (77\% of the total EMUs); however, she also used text box (13\% of total EMUs) and speak button EMUs (10\% of total EMUs). Ellen experienced trouble sources on $9 \%$ of total EMUs; however, she repaired the majority of these breakdowns (i.e., 86\%) with an average trajectory of 6 EMUs. Ellen strongly agreed (i.e., 5) that the LDs helped her tell her story and agreed (i.e., 4) that the words helped her tell her story.

LD without text boxes. During this retell, she used spoken (72\% of total EMUs), drawn (10\% of total EMUs), picture ( $9 \%$ of total EMUs), written (5\% of total EMUs), and speak button EMUs (4\% of total EMUs). Trouble sources resulted from $9 \%$ of Ellen's total EMUs, of which $44 \%$ were repaired with an average trajectory of 10 EMUs. Ellen neither agreed nor disagreed (i.e., 3) that the LDs were helpful; however, she strongly agreed (i.e., 5) that the words would have helped her tell her narrative.

\section{Jack}

Jack was a 57-year-old male who was 48 months post-CVA. At the time of his stroke, he was a retired business manager and avid traveler. He remained active in the community as an advocate for stroke survivors and volunteered at a local rehabilitation hospital. Jack had received physical, occupational, and speech rehabilitation services. When asked, he stated that he was familiar with AAC (through observations at stroke support groups); however, he had never used a high-technology device. Jack exhibited moderate Broca's aphasia (i.e., WAB-R AQ = 61.8; Kertesz, 2007) and communicated primarily through the use of residual speech characterized by reduced phrase length (e.g., 3-5 words), decreased word retrieval, and fillers.

Figure 4 illustrates Jack's use of the available EMUs across the four conditions and Figure 5 displays his trouble source and repair data. Table 2 displays Jack's perceived helpfulness of the visual and linguistic supports. Jack rated the helpfulness of the AAC device as very helpful when retelling his narratives after his final retell (Table 2).

PR photographs with text boxes. Jack used a variety of EMUs to retell his narrative in the PR + TB condition. He used spoken ( $85 \%$ of the total EMUs), speak button ( $9 \%$ of the total EMUs), written ( $4 \%$ of the total EMUs), text box ( $1 \%$ of the total EMUs), and picture EMUs (1\% of the total EMUs). During this retell, $5 \%$ of his EMUs resulted in trouble sources, of which Jack was able to repair $25 \%$ with an average trajectory of 5 EMUs. Jack agreed that the PR photographs were helpful (i.e., 4) and strongly agreed (i.e., 5) the words were helpful.

PR photographs without text boxes. Jack expressed himself with spoken (69\% of the total EMUs), picture (19\% of the total EMUs), speak button (8\% of the total EMUs), and written EMUs (4\% of the total EMUs). He experienced trouble sources on $8 \%$ of the total EMUs and successfully repaired $50 \%$ of these breakdowns. The average trajectory of Jack's trouble sources was 7 EMUs. He strongly agreed (i.e., 5) that the pictures helped and also agreed (i.e., 4) that the words would have helped him tell his narrative. Jack demonstrated a relatively high amount

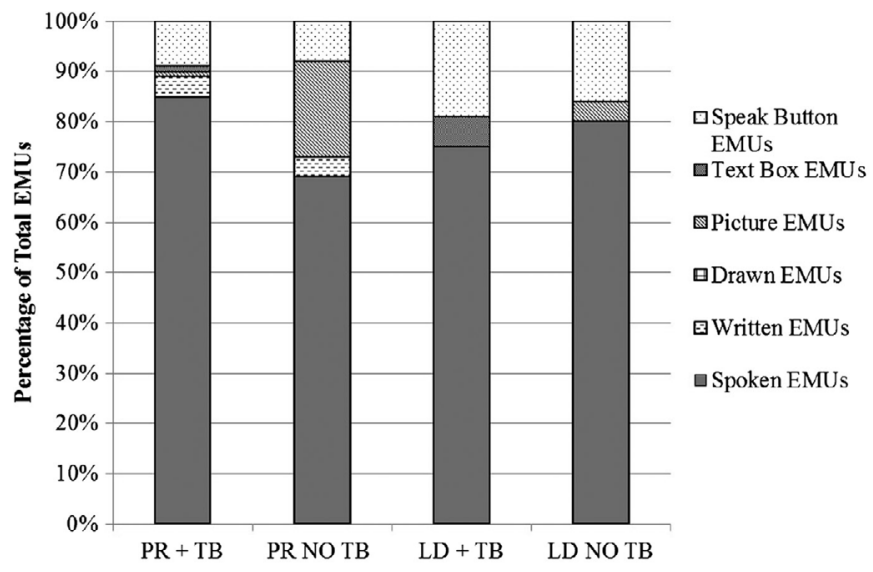

Figure 4. The percentage of expressive modality types out of the total expressive modality units Jack used during each narrative retell condition. 


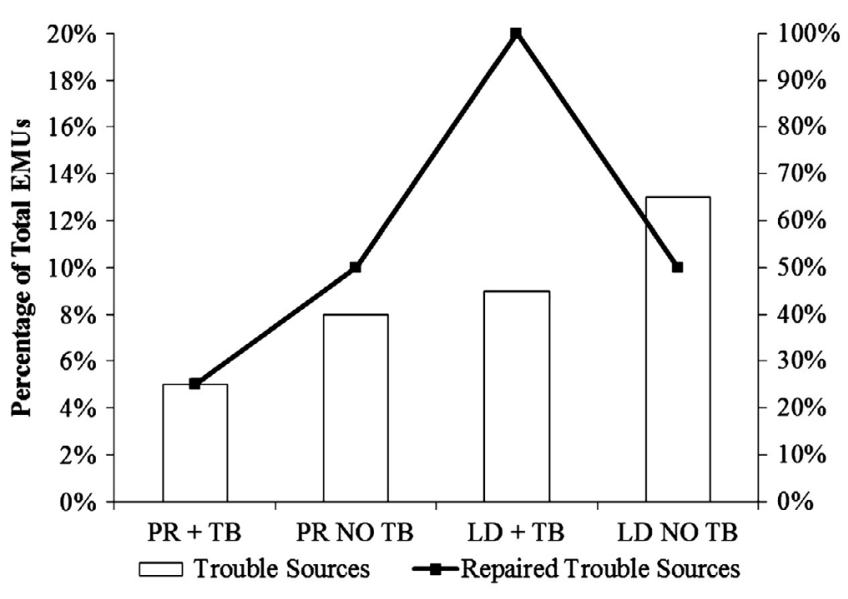

Figure 5. The percentage of trouble sources Jack experienced out of the total amount of EMUs in each condition as well as the percentage of trouble sources Jack was able to repair.

of picture EMUs in this condition and further displayed a preference for visual supports over linguistic supports during the interview with the researcher as shown below:

\section{Researcher: Was it better this time?}

Jack: Yeah, better more places and names.

Researcher: What if there were words here [pointing to the

PR NO TB interface]? Would that have helped you?

Jack: Yeah :03 sec but pictures.

Researcher: More pictures would have been ideal?

Jack: Yes, helpful.

LD with text boxes. Jack used few types of EMUs to tell his narrative in the LD + TB condition: spoken (75\% of total EMUs), speak button (19\% EMUs), and text box EMUs (6\% of the total EMUs). Trouble sources resulted from $9 \%$ of his total EMUs; yet, Jack successfully repaired $100 \%$ of these breakdowns with an average trajectory of 3 EMUs. He strongly agreed (i.e., 5) that both the LDs and words helped him tell his story.

$L D$ without text boxes. Jack relied heavily on spoken EMUs to retell his narrative in the LD NO TB condition, which was composed of $80 \%$ spoken EMUs. He also used speak button (16\% of total EMUs) and picture EMUs ( $4 \%$ of total EMUs). Jack experienced the highest rate of trouble sources in this condition at $13 \%$ of the total EMUs; he was able to repair $50 \%$ of these breakdowns with an average trajectory of 3 EMUs. Jack agreed (i.e., 4) that the words would have helped him retell his narrative. He also agreed (i.e., 4) that the pictures helped even though he reported difficulty relating the LDs to his narrative. During his retell, he stated, "Really, uh, this one [points to a LD] I don't know what that for."

\section{Sarah}

At the time of the study, Sarah was a 42-year-old female who was 81 months poststroke. She returned to work- although not to her prestroke position-at a local nonprofit organization and enjoyed being a full-time mother to her two young children. Sarah received speech-language therapy services earlier in her recovery and occasionally used a Palmtop ${ }^{\mathrm{TM}}$ high-technology AAC device. Typically, her AAC interactions involved her demonstrating the device; however, she did not frequently use it in a functional manner. She presented with moderate Broca's aphasia (i.e., $\mathrm{WAB}-\mathrm{RAQ}=53.9$; Kertesz, 2007) and communicated primarily with moderately apraxic speech of one- to two-word phrases, which she supplemented with gestures and writing.

Sarah's use of EMUs during the four narrative retell conditions is displayed in Figure 6, and her trouble source data are presented in Figure 7. Table 2 presents Sarah's perceived helpfulness of the visual and linguistic supports. Overall, she rated the helpfulness of the AAC device as very helpful when retelling her narratives (Table 2).

PR photographs with text boxes. Sarah used a variety of modalities to retell her narrative in the PR + TB condition. Her narrative was composed of spoken (69\% of the total EMUs), text box (16\% of the total EMUs), picture (11\% of the total EMUs), drawn (3\% of the total EMUs), and speak button EMUs (1\% of the total EMUs). Sarah experienced the fewest trouble sources (4\% of total EMUs) during this condition and repaired $67 \%$ of these breakdowns with an average trajectory of 6 EMUs. She strongly agreed (i.e., 5) that the PR photographs were helpful and agreed (i.e., 4) that the words helped her tell the narrative.

PR photographs without text boxes. Sarah relied heavily on spoken EMUs (73\% of the total EMUs) to tell her narrative in the PR NO TB condition. She also used speak button $(11.5 \%$ of the total EMUs), picture (11.5\% of the total EMUs), and written EMUs (4\% of total EMUs). Sarah demonstrated the highest rate of trouble sources during this narrative retell ( $9 \%$ of total EMUs). She repaired $67 \%$ of her trouble sources with an average trajectory of 6 EMUs. Sarah strongly agreed (i.e., 5) that the PR photographs were helpful and agreed (i.e., 4) that the words would have helped her retell her story.

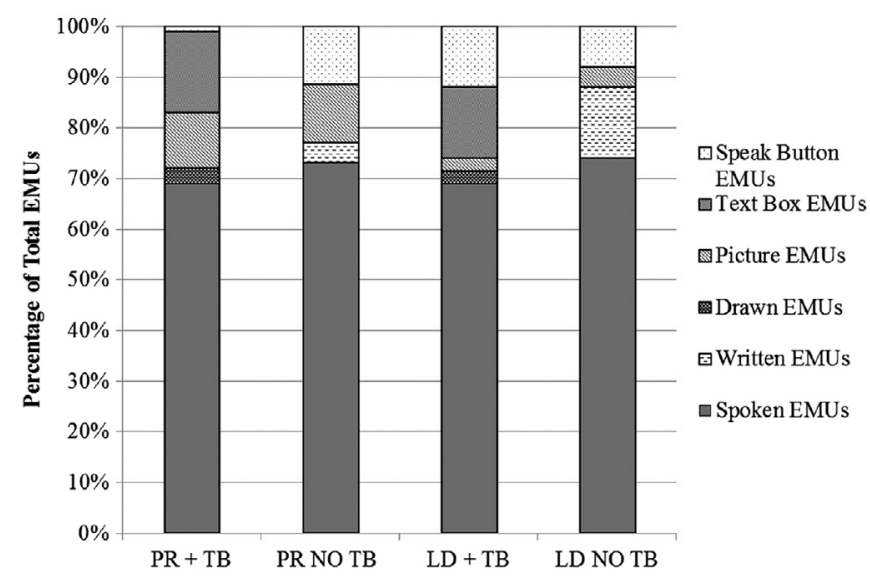

Figure 6. The percentage of expressive modality types out of the total expressive modality units Sarah used during each narrative retell condition. 


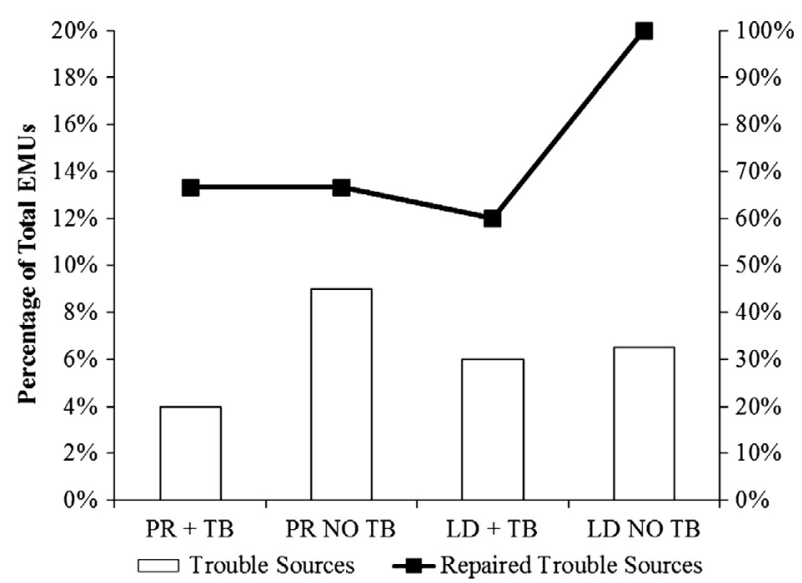

Figure 7. The percentage of trouble sources Sarah experienced out of the total amount of EMUs in each condition as well as the percentage of trouble sources Sarah was able to repair.

LD with text boxes. Sarah used spoken (69\% of total EMUs), text box (14\% of total EMUs), speak button (12\% of total EMUs), picture (2.5\% of total EMUs), and drawn EMUs (2.5\% of total EMUs) to tell her narrative in the LD + TB condition. Trouble sources stemmed from $6 \%$ of her total EMUs, of which she repaired $60 \%$ with an average trajectory of 12 EMUs. When prompted to comment on the helpfulness of the pictures and text, Sarah agreed (i.e., 4) that the LDs helped and strongly agreed (i.e., 5) that the words helped her tell her narrative. Sarah continued to describe how the text helped her retell the narrative in the subsequent exchange:

Researcher: Were the written words helpful?

Sarah: Words yes um :03 sec talking no.

Researcher: So it helped when the words wouldn't come out that you could point here [pointing to the text boxes on the LD + TB interface]?

Sarah: Yeah [pointing to the text boxes].

LD without text boxes. Sarah relied mostly on spoken $(74 \%$ of total EMUs) and written EMUs (14\% of total EMUs) to retell her story. She also used speak button (8\% of total EMUs) and picture EMUs ( $4 \%$ of total EMUs). Sarah experienced trouble sources on $7 \%$ of EMUs, of which $100 \%$ were repaired with an average trajectory of 6 EMUs. She agreed (i.e., 4) that the pictures helped and strongly agreed (i.e., 5) that the words would have helped tell her story.

\section{Claire}

Claire was a 70-year-old female homemaker who was 42 months post-CVA at the time of the study. She was a wife, mother, grandmother, and an active member of her local church. Claire had no prior experience with high-technology AAC devices. She received physical, occupational, and speech and language therapies after her stroke and was not receiving treatment at the time of this project. She demonstrated moderate Broca's aphasia (i.e., WAB-R AQ = 64.9; Kertesz, 2007) and primarily communicated through mildly dysarthric and apraxic speech characterized by reduced phrase length (e.g., 3-5 words), imprecise articulation, and slow rate of speech, which she supplemented with gestures.

The pattern of Claire's EMU usage is illustrated in Figure 8, and her trouble source and repair data are depicted in Figure 9. Claire's perceived helpfulness of the visual and linguistic supports and AAC device is displayed in Table 2. At the end of her final retell, she rated the helpfulness of the AAC device as very helpful when retelling her narratives (Table 2).

$P R$ photographs with text boxes. Claire used a combination of spoken (64\% of total EMUs), text box (15\% of total EMUs), speak button (11\% of total EMUs), and picture EMUs (10\% of total EMUs) to retell this story. Claire experienced the fewest trouble sources in the PR + TB condition (i.e., $3 \%$ of the total EMUs), and she repaired $100 \%$ of her trouble sources with an average trajectory of 5 EMUs. Claire strongly agreed (i.e., 5) that the PR photographs and agreed (i.e., 4) that the words helped her tell her story.

PR photographs without text boxes. Claire relied heavily on spoken (64\% of the total EMUs) and picture EMUs (28\% of total EMUs) to retell her narrative. She also used speak buttons (6\% of EMUs) and writing (3\% of total EMUs). Claire experienced trouble sources on $6 \%$ of her total EMUs and repaired 38\% of these breakdowns with an average trajectory of 5 EMUs. When asked to judge the helpfulness of the PR photographs, Claire strongly agreed (i.e., 5) that they helped her tell her story. She was neutral (i.e., 3) as to whether the presence of words would have helped her tell her story.

LD with text boxes. During the LD + TB narrative retell condition Claire used a combination of spoken (67\% of total EMUs),

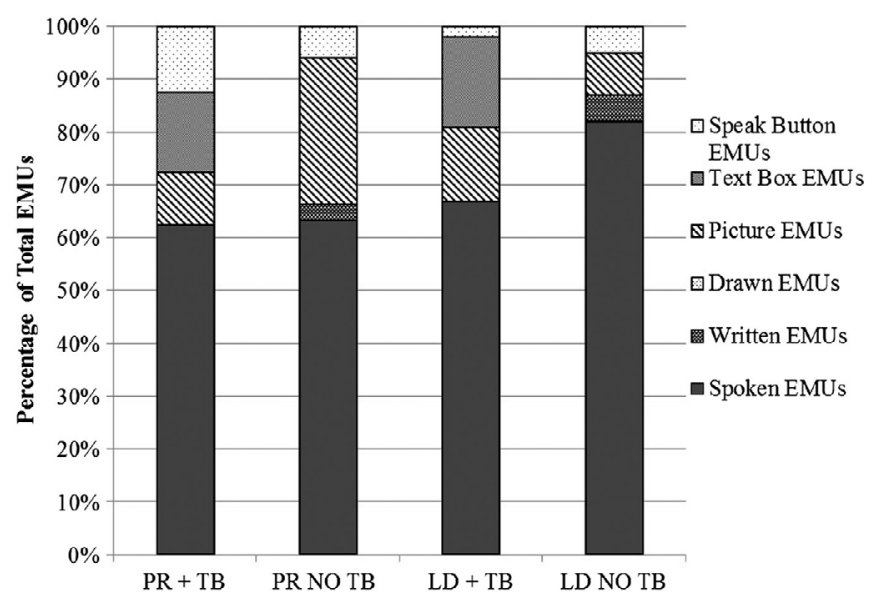

Figure 8. The percentage of expressive modality types out of the total expressive modality units Claire used during each narrative retell condition. 


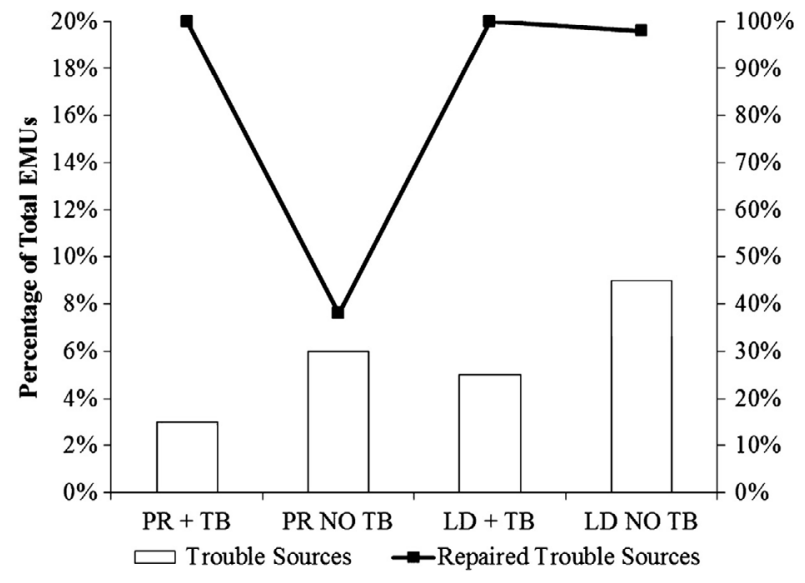

Figure 9. The percentage of trouble sources Claire experienced out of the total amount of EMUs in each condition as well as the percentage of trouble sources Claire was able to repair.

text box (17\% of total EMUs), picture (14\% of total EMUs), and speak button EMUs (2\% of total EMUs). Claire repaired 100\% of her trouble sources (5\% of the total EMUs) with an average trajectory of 6 EMUs. She strongly agreed (i.e., 5) that both the LDs and words helped her tell the story.

$L D$ without text boxes. Claire used a variety of EMUs to share her narrative. Specifically, she relied on spoken $(82 \%$ of total EMUs) as well as pictures (8\% of total EMUs), writing (5\% of total EMUs), and speak button EMUs (5\% of total EMUs). Claire had the highest rate of trouble sources ( $9 \%$ of total EMUs) during this narrative retell. However, she was able to repair $89 \%$ of her breakdowns with an average trajectory of 6 EMUs. Claire strongly agreed (i.e., 5) that the LDs were helpful when retelling her narrative and that the presence of words would have helped her retell her narrative. Although Claire agreed that the LDs were helpful, she stated that she preferred the PR photographs during the interview in the following exchange:

Researcher: So the pictures help? [gesturing to the displayed LD NO TB condition]

Claire: Yeah, yes but:02 sec. [shaking head no while pointing to the LD pictures]

Researcher: But you would prefer your own?

Claire: Yes.

\section{Discussion}

Three main findings warrant further discussion: (a) the types of expressive modalities used by the participants, (b) the overall low incidence of trouble sources, and (c) the participants' perceived helpfulness of the AAC device and interface elements. The following sections summarize these results and discuss how they may be interpreted in light of the case series design. The researchers also offer suggestions for future work in the area of AAC interface design for people with aphasia. Clinical implications related to the use of VSDs with people who have aphasia are also provided and generate a springboard for future research in AAC aphasiology. Because this case series describes the communicative behavior of four college graduates from Mid-Western American Caucasian culture, clinicians should consider the background and educational experiences of the people with aphasia whom they serve when applying any clinical findings.

\section{Communicative Use of EMUs}

All of the participants expressed themselves predominately through spoken EMUs, which strengthens the idea that hightechnology AAC devices do not hinder the desire or ability to use spoken language (Dietz et al., 2012). While the presence of AAC did not appear to impede spoken productions, it is unknown whether the spoken EMUs used translated into improved linguistic performance in the presence of AAC. These findings warrant work that examines the relationship of AAC use, or treatment, on the quality and the quantity of spoken discourse produced by people with aphasia.

After spoken EMUs, the participants tended to utilize hightechnology VSD EMUs (i.e., picture, text box, and speak button EMUs) more often than low-technology AAC EMUs (i.e., written and drawn EMUs) to retell their narratives. This finding may reflect the challenges many people with aphasia experience writing or drawing secondary to hemiparesis or concomitant limb apraxia. It is equally plausible that the participants experienced more efficient and effective communication when they referenced the VSDs, which is congruous with previous research on the utilization of augmented input to foster communication (Garrett \& Beukelman, 1995; Garrett \& Huth, 2002; Wallace et al., 2012). Compared to the LD retells, the people with aphasia in this study demonstrated a higher frequency of picture EMUs during the PR retells to facilitate information transfer. This result may be due to the preference many people with aphasia have for interacting with their own materials (Dietz et al., 2012; McKelvey et al., 2010).

Along with picture EMUs, text box EMUs emerged as a preferred method of communication for the participants. When available, three of the four people with aphasia utilized text box EMUs regularly. The case-series design revealed that one participant's behavior (i.e., Jack) was in contrast to the other participants' behavior. Specifically, he referenced the text infrequently and tended to use more spoken and speak button EMUs. Yet, the other participants showed a propensity to supplement their narrative retells with written EMUs when text boxes were not available, revealing that the participants used and relied on linguistic supports to tell their narratives. Further, it stands to reason that when text was not available to be referenced, the people with aphasia wrote to add pertinent information or to ensure that the listener understood their narratives. The listener in this investigation may also have benefitted from the presence of text as Dietz et al. (2012) reported that a communication partner relied heavily on text to be confident in her understanding of narratives told by people with aphasia. 


\section{Trouble Sources and Repairs}

Generally speaking, the participants experienced low rates of trouble sources, regardless of the type of visual and linguistic supports included on the VSDs. Similar to findings by Dietz et al. (2012), the low rate of communicative breakdowns may be related to the dynamics of the structured communication setting. That the people with aphasia consented to participate in a research project may have changed the task of retelling a narrative to a type of "institutional discourse" or "a type of talk in which the rights of the SLT [speech-language therapist] to introduce topics, apportion turns, and pursue responses are accepted in advance" (Lindsay \& Wilkinson, 1999, p. 306). Even more, the listener in this study was a young woman with no prior experience conversing with people with aphasia and was charged by the researchers with the task of listening. As such, out of respect for her elders and in attempt to adhere to the research instructions, she may have not asked for clarification or more information when she misunderstood portions of the participants' retells. Because the retells became a form of institutional discourse with an appointed listener, the participants may have felt that it was the duty of the listener to pursue clarification and initiate repairs of communicative breakdowns. Therefore, this perception may be reflected in the low rate of trouble sources experienced across participants. Changing the narrative retell task to be more like that of a conversation with a peer or a family member in which conversational turns, topics, and repairs are negotiated throughout the exchange and not predetermined as in institutional discourse may reveal changes in the EMUs used and the breakdowns experienced and repaired (Cherney, 1998; Lindsay \& Wilkinson, 1999). Because communication is influenced by audience (Cherney, 1998), future research endeavors should explore how trouble sources and repair sequences may differ when people with aphasia use AAC to interact with various types of communication partners (e.g., peers, family members, authority figures).

While noting the relatively low rates of trouble sources, it is important to consider that the people with aphasia had four interactive opportunities (i.e., one session) and the listener had 16 opportunities (i.e., four sessions) across the duration of the study. As such, it is possible that her naiveté about aphasia and how to interact with people with aphasia decreased over time, which could have influenced the success of the participants telling their stories. In this study, the listener complied with the interaction guidelines $95 \%$ of the time, and anecdotally, the researchers observed no qualitative changes in how she requested or verified information with the participants. Therefore, her behavior did not likely impact the number of trouble sources experienced, percentage of trouble sources repaired, or the trajectory of the trouble sources.

That the participants in the study experienced similar and relatively low rates of communicative breakdowns regardless of the type of visual support presented offers data to support the growing evidence that people with aphasia are able to successfully use PR photographs to augment their communication (Dietz et al., 2006, 2012; McKelvey et al., 2007, 2010). The Dietz et al. (2012) study revealed that the presence of nonpersonally relevant photographs generated off-topic discourse (i.e., communicating that the photographs were not theirs) and trouble sources for people with aphasia. Perhaps the participants in the current study did not feel compelled to communicate that the LDs were not theirs, because LDs are clearly nonpersonally relevant. As such, the LDs did not generate any off-topic discourse. Thus, LDs may be a more acceptable alternative to nonpersonally relevant photographs when PR photographs are not available, at least when retelling narratives. The presence of text appeared to have a positive influence on the number of trouble sources exhibited. With the exception of Ellen, the participants experienced slightly fewer trouble sources when text was available. This finding adds to the current literature to support the incorporation of text into AAC systems for people with aphasia (Dietz et al., 2006, 2012; Garrett \& Beukelman, 1995; Garrett \& Huth, 2002).

Surprisingly, the type of visual and linguistic supports did not appear to affect the rate of trouble sources repaired. In fact, each participant demonstrated a unique pattern of repair sequences and trajectories (e.g., length of the repair sequence). However, text seemed to facilitate the repair of trouble sources for one participant (i.e., Claire), as she was able to repair $100 \%$ of her breakdowns in the text box conditions. It is interesting to note that Claire also displayed seemingly intact basic reading abilities with a perfect score on the reading comprehension of sentences subtest of the WAB-R (Kertesz, 2007). The unique patterns of repair behavior could be because of the lack of training given to the participants on how to exploit visual and linguistic supports to repair communication breakdowns. This investigation only allowed the participants one opportunity to interact with the various VSD interfaces to retell their narratives, and people with aphasia may display trends when repairing trouble sources with repeated exposure and training (Koul et al., 2005). As such, it is imperative that future research emphasize strategic and social competence or instruction that teaches people with aphasia how to use elements of AAC VSD interfaces to repair communicative breakdowns. In particular, the relationship between the linguistic profiles of people with aphasia (i.e., stronger reading ability) and successful repair sequences could inform the creation of AAC training paradigms (i.e., how to use text to repair trouble sources).

\section{Perceived Helpfulness of Supports}

The participants in this study reported that both the PR photographs and LDs were equally helpful when retelling narratives. Two participants (i.e., Ellen and Jack) even stated that they would like more than two images incorporated into the interface designs. As such, the number and types of visual supports that people with aphasia prefer to be incorporated into VSD interfaces is an area that requires closer examination. Even more compelling is that the people with aphasia used PR photographs at a slightly higher rate than the LDs when available, which is consistent with the theme in AAC and aphasia literature that people with aphasia display a preference to use PR 
materials (e.g., Dietz et al., 2012; McKelvey et al., 2010; Smith \& Garrett, 2005). On the basis of the participants' perceived helpfulness, PR photographs should be incorporated into VSD interface designs when possible, especially for narrative retells. It is feasible that the type of visual supports people with aphasia find most helpful may change for different types of communication and across cultures. For example, some people with aphasia may find LDs to be more helpful than PR photographs when sharing basic needs but may find PR photographs more supportive when socializing. Therefore, it is important for future investigations to compare and contrast the perceived helpfulness of various types of visual supports across multiple communication tasks and communication partners.

It is also noteworthy that the participants with no prior AAC experience (i.e., Ellen, Jack, and Claire) appeared comfortable using high-technology AAC to augment their narrative retells even without training. In fact, all of the participants stated that they found the AAC device to be either helpful or very helpful. However, researcher bias may have influenced how the participants reported their perceptions of the helpfulness of the images, text, and AAC device. Because the first author facilitated an informal interview with all the participants on their perceived helpfulness of the various interfaces, it is possible that she may have unwittingly steered the conversations to result in favorable ratings and comments about using AAC. Also, the participants may have reported what they thought the researcher would like to hear, since they knew one of the purposes of the study was to investigate the helpfulness of AAC devices. Therefore, true preferences and perceived helpfulness of AAC devices and interface options may be better estimated if a blinded researcher completes the interview. Accurately determining the perception and preferences of people with aphasia is an important next step in integrating AAC into the rehabilitation process. This type of data should be collected following intervention studies that allow people with aphasia to use AAC across a period of time to communicate on a wide array of topics, with a range of different people, in a variety of settings.

\section{Clinical Implications}

Technology and communication applications are more readily available than ever before. People with aphasia are exploiting communication applications on various platforms (e.g., iPads, tablets, AAC systems) to augment their communication (Brandenburg et al., 2013; Dietz et al., 2011, 2012; McNaughton \& Light, 2013). Furthermore, technological advances have made the inclusion of PR photographs, LDs, and text effortless. The knowledge of interface features that elicit the most effective and efficient communication for many people with aphasia is emerging. This study provides additional data to suggest that PR photographs and text boxes are perceived as helpful by some people with aphasia when retelling narratives. Nevertheless, the participants in this investigation also reported LDs to be helpful. Because LDs did not appear to cause trouble sources any more frequently than PR photographs, LDs may serve as an appropriate visual support when PR photographs are unavailable. Still, individual assessment is necessary to determine the optimum type and combination of visual and linguistic supports for people with aphasia when implementing AAC. Ideally, speech-language pathologists and people with aphasia will capitalize on advances in technology to customize interfaces that meet the needs of various communication situations and partners.

Acknowledgment - Portions of this article were presented at the 2013 Clinical Aphasiology Conference in Tucson, AZ, and the 2013 Annual Convention of the American Speech-Language-Hearing Association in Chicago, IL. We thank Brittany Sileo Fehskens, Bridget Brown, Devan Macke, and Jennifer Keelor for their contributions to this project. We are also grateful for the participants' time and effort.

Disclosure - The authors have declared that no competing interests existed at the time of publication.

\section{References}

Beukelman, D. R., \& Mirenda, P. (2013). Augmentative and alternative communication: Supporting children and adults with complex communication needs. Baltimore, MD: Brookes.

Brandenburg, C., Worrall, L., Rodriguez, A. D., \& Copland, D. (2013). Mobile computing technology and aphasia: An integrated review of accessibility and potential uses. Aphasiology, 27, 444-461.

Cherney, L. (1998). Pragmatics and discourse: An introduction. In L. R. Cherney, B. B. Shadden, \& C. A. Coelho, eds., Analyzing discourse in communicatively impaired adults (pp. 1-7). Gaithersburg, MD: Aspen Publishers.

Creswell, J. W. (2009). Research design qualitative, quantitative, and mixed methods approaches. Los Angeles, CA: Sage.

Dietz, A., Ball, A., \& Griffith, J. (2011). Reading and writing with aphasia in the 21st century: Technological applications of supported reading comprehension and written expression. Topics in Stroke Rehabilitation, 18, 758-769.

Dietz, A., Beukelman, D. R., \& McKelvey, M. (2006). Visual scene display (VSD): New AAC interfaces for persons with aphasia. Perspectives on Augmentative and Alternative Communication, 15, 13-17.

Dietz, A., Hux, K., McKelvey, M. L., Beukelman, D. R., \& Weissling, K. (2009). Reading comprehension by people with chronic aphasia: A comparison of three levels of visuographic contextual support. Aphasiology, 23, 1053-1064.

Dietz, A., Weissling, K., Griffith, J., \& McKelvey, M. (2012, July-August). Personalizing AAC for people with aphasia: The role of text and visuographic supports. Seminar presented at the 2012 International Society for Augmentative and Alternative Communication (ISAAC), Pittsburgh, PA.

Elman, R. J., \& Larsen, S. (2010, May). Computer and Internet use among people with aphasia. Paper presented at the Clinical Aphasiology Conference, Isle of Palms, SC.

Fox, L., Sohlberg, M. M., \& Fried-Oken, M. (2001). Effects of conversational topic choice on outcomes of an augmentative communication intervention for adults with aphasia. Aphasiology, 15, 171-200.

Garrett, K., \& Beukelman, D. R. (1995). Changes in interaction patterns of an individual with severe aphasia given three types of partner support. Clinical Aphasiology, 23, 237-251.

Garrett, K. L., \& Huth, C. (2002). The impact of graphic contextual information and instruction on the conversational behaviors of a person with severe aphasia. Aphasiology, 16, 523-536. 
Kertesz, A. (2007). Western Aphasia Battery-Revised. San Antonio, TX: Pearson Education.

Koul, R., Corwin, M., \& Hayes, S. (2005). Production of graphic symbol sentences by individuals with aphasia: Efficacy of a computerbased augmentative and alternative communication intervention. Brain and Language, 92, 58-77.

Lindsay, J., \& Wilkinson, R. W. (1999). Repair sequences in aphasic talk: A comparison of aphasic speech and language therapist and aphasic-spouse conversations. Aphasiology, 13, 305-325.

McKelvey, M., Dietz, A., Hux, K., Weissling, K., \& Beukelman, D. R. (2007). Performance of a person with chronic aphasia using a visual scene display prototype. Journal of Medical Speech-Language Pathology, 15, 305-317.

McKelvey, M., Hux, K., Dietz, A., \& Beukelman, D. R. (2010). Impact of personal relevance and contextualization on word picture matching by people with aphasia. American Journal of Speech-Language Pathology, 19, 22-33.

McNaughton, D., \& Light, J. (2013). The iPad and mobile technology revolution: Benefits and challenges for individuals who require augmentative and alternative communication. Augmentative and Alternative Communication, 29, 107-116.
McNeil, M. R., \& Pratt, S. R. (2001). Defining aphasia: Some theoretical and clinical implications of operating from a formal definition. Aphasiology, 15, 901-911.

Smith, C. E., \& Garrett, K. L. (2005, November). Comprehension of contextual vs. decontextual written choices in severe aphasia. Poster presented at the Annual American Speech-Language- Hearing Association Convention, San Diego, CA.

True, G., Bartlett, M. R., Fink, R., Linebarger, M., \& Schwartz, M. (2010). Perspectives of persons with aphasia towards Sentence Shaper To Go: A qualitative study. Aphasiology, 24, 1032-1050.

Wallace, S., Dietz, A., Hux, K., \& Weissling, K. (2012). Augmented input: The effect of visuographic supports on the auditory comprehension of people with chronic aphasia. Aphasiology, 26, 162-176.

Wallace, S., \& Hux, K. (2014). Effect of two layouts on high technology AAC navigation and content location by people with aphasia. Disability and Rehabilitation: Assistive Technology, 9, 173-182. do i:10.3109/17483107.2013.799237 